\title{
Analysis and Optimization of Wind Farm Performances
}

\author{
Stefano Leonardi \\ Dept. Mech. Eng., the University of Texas at Dallas \\ USA
}

\begin{abstract}
In recent years, the wind energy industry has experienced a considerable technological and economic expansion leading to the design of large and dense power plants. As the turbines are being placed closer together, in order to minimize capital costs, the overall efficiency is being affected by wake interaction between upstream and trailing turbines.

With blade lengths of the order of the order of $100 \mathrm{~m}$, the flow field in a wind turbine array is inherently coupled to unsteady dynamics at the synoptic scale. To assess performances and optimize the power production of wind farms we developed a numerical framework coupling the Weather Research and Forecasting (WRF) Model, a meso-scale numerical weather prediction model and our in-house LES code to retain the large-scale variability and resolve, in the limit of LES resolution, the turbine wakes. Results agree well with LiDAR measurements and SCADA data and provide accurate values for the transient load variations in the rotor disk. The digital wind farm model is then used to test control algorithms aimed at optimizing the power production of the plant. In particular, with Extremum-Seeking Control (ESC) on torque gain and yaw angle of the rotor disk, we were able to increase the power of the array of turbines of up to 7-10\%. To assess the impact on the overall Annual Energy Production we developed a surrogate model combining or numerical simulations with generalized Polynomial Chaos1 (gPC). With gPC, the response function of the wind farm power production to the input parameters (i.e. wind speed $U$ and direction $\theta$ ) is projected onto a polynomial (truncated at order $N$ ) in the parameter space. The $\mathrm{N}$ coefficients of the polynomial expansion are determined using a set of $\mathrm{N}$ high fidelity simulations for specific wind speeds and directions. Therefore, gPC allows obtaining an accurate surrogate model (which consists in the polynomial expansion) with a relatively limited number $\mathrm{N}$ of large-eddy simulations. The surrogate model can be used to calculate Annual Energy Production and assess the risk of investment or repowering.
\end{abstract}

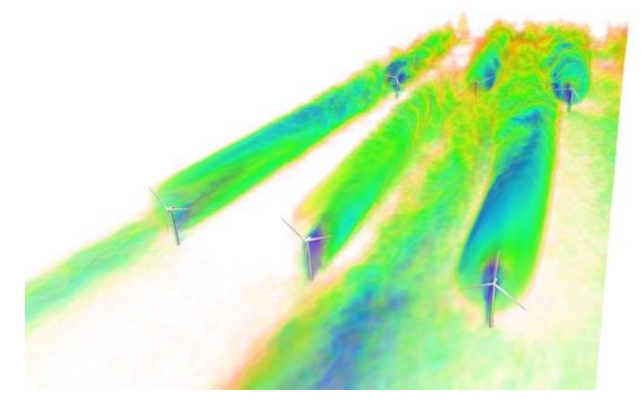

Figure 1 Color contours of instantaneous velocity 